\title{
Addition of Glutamine in the Human Platelets Could Prevent Toxic Effect of Ultraviolet-C Induced Platelets Activation
}

\author{
Mazhar Mushtaq \\ Basic Medical Sciences Department, Sulaiman Al Rajhi University, Al-Bukayriah, Saudia Arabia
}

Email address:

dr_hcg@yahoo.com

\section{To cite this article:}

Mazhar Mushtaq. Addition of Glutamine in the Human Platelets Could Prevent Toxic Effect of Ultraviolet-C Induced Platelets Activation. Biomedical Sciences. Vol. 7, No. 1, 2021, pp. 36-40. doi: 10.11648/j.bs.20210701.17

Received: March 1, 2021; Accepted: March 17, 2021; Published: March 26, 2021

\begin{abstract}
The primary function of platelets is to prevent bleeding. Transfusion of platelets can be prophylactic or therapeutic. From the storage perspective of blood and blood components, standard protocols are followed around the world to ensure the safe operation of blood banks. The Use of UV-C light in treating platelets has become a valuable method for storage and efficacy of platelets concentration in the blood bank. However, its deleterious effect remains, such as activation of platelets, thus losing their physiological function. In this study we intend to demonstrate that addition of glutamine in the platelets concentrate could prevent the toxic effect of UV-C. This study was conducted using human or mouse platelets. Use of positive and negative control in all experiment were ensured to validate the findings. In vitro thrombus formations was assessed before and after exposure of platelets to appropriate dose of UV-C. To assess the cytotoxic effect of $50 \mathrm{mM}$ of glutamine on the platelets, methylthiazoletetrazolium (MTT) was used to validate the viability effect. We have successfully demonstrated that physiological damages done during the exposure of platelet concentrate to UV-C could be alleviated by the use of glutamine. Our study demonstrated that the toxic effect of UV-C on the platelets could be preserved by adding appropriate concentration of glutamine.
\end{abstract}

Keywords: Platelets, Glutamine, UV-C, Thrombus Formation

\section{Introduction}

Platelets are tiny cellular fragments of large bone marrow cells (megakaryocytes). They make up only a minute fraction of the total blood volume. The primary function of platelets is to prevent bleeding.

Various pathological disorders are attributed to their numbers and functions. Many factors have been documented in literature regarding the root cause of the decrease in number or functions of platelets. Frequently, such individuals are transfused with platelets to maintain the hemostasis.

The use of broad-spectrum wavelength Mirasol $^{\mathrm{TM}}$ Pathogen Reduction Technology System of ultraviolet (UV) light (UV-A (60\%), UV-B (100\%), and UV-C (20\%)) and beneficial effect of UV-C on the platelet concentrate has been well documented $[1,2]$. However, its pitfall remains, such as decrease in the physiological function, [3] and generation of ROS [4]. Other biomolecules that are altered/produced during the exposure of platelets concentrate are; lactic acid production, P-selection exposure and phosphatidylserine exposure [3]. In an interesting study, it was demonstrated that UV-C pathogen inactivation treatment does not influence the activation of integrin's of the platelets; however, it mitigates thrombus formation property of platelets, when subjected to micro fluidic flow chamber [5]. Such a decrease of physiological function leaves the platelets, inevitably of nouse, if they are to be transfused, especially for those who are suffering from severe platelet disorders.

In a very interesting study conducted by Murphy et al., they estimated the level of 17 plasmas' amino acid during the storage of platelet concentrate and concluded that the concentration of 16 amino acid remained unchanged, whereas the concentration of glutamine fell to zero by day four [6].

In this study, we intend to demonstrate that the addition of glutamine in the human platelets concentrate could prolong 
the physiological function. We, used $50 \mathrm{mM}$ concentration of L-glutamine in freshly prepared human or mouse platelets and they were exposed to UV-C light for short intervals.

\section{Materials and Methods}

\subsection{Reagents}

Thrombin, L-Glutamine (Glutamine). CCK8 Kit from Dijindo Laboratories and various other reagents from SigmaAldrich.

\subsection{Platelet Preparation}

Male mice, C57BL/6, 4 to 6 weeks, were inbred and maintained at the animal house of the university. All animal studies were performed according to a protocol approved by the institutional animal care of Chonbuk National University Medical School. Blood was drawn by cardiac puncture into Acid Citrate Dextrose (ACD) (20mM citric acid, $110 \mathrm{mM}$ sodium citrate, and $5 \mathrm{mM}$ glucose) 1:10 ratio V/V. Platelets were prepared as previously described [7]. Platelets in Tyrode Buffer (TB) or Platelets Rich Plasma (PRP) were then allowed to settle for 60 minutes at $37^{\circ} \mathrm{C}$, before their use for the experiment. For the calcium study, $0.02 \mathrm{U}$ of apyrase was added during the resting period to minimize the effect of ADP and ATP. Calcium was added if stated, else calcium-free buffer was used.

Human platelets were prepared using the same protocol with phlebotomy after informed consent was obtained from the participants, as per guideline of the Biochemistry department. Participants were explained the purpose of it. The participants were also screened before for the potential risk assessment, and essential safety procedures were followed approved by hospital laboratory.

\subsection{UV-C Irradiation}

The appropriate volume and concentration of platelets with or without glutamine were irradiated from the above in 96 or 24-well plates, using Vilber Lourmat BLX-254 emitting at $254 \mathrm{~nm}$. According to the company's specification, platelets were irradiated with a constant intensity of 0.5 joules for 100 seconds at room temperature, with continuous shaking in an uncovered petri-dish of $60 \times 15 \mathrm{~mm}$.

Since Gravemann et al. had demonstrated that the use of 0.2 joules of UV-C in Theraflex ${ }^{\mathrm{TM}}$ causes minimal damage to $\alpha \mathrm{IIb} \beta 3$ and 0.6 joules of UV-C causes significant damage to integrin [8], we used 0.5 joules of UV-C to demonstrate that maximum exposure of platelets to UV-C, which can cause significant changes to integrin, could be ameliorated by the use of glutamine.

\subsection{In vitro Thrombus Formation}

Platelet were stimulated with thrombin $0.5 \mathrm{U} / \mathrm{ml}$ or exposed to UV-C with or without glutamine under continuous shaking After the desired time, extra platelets were aspirated and the thrombus formed at the bottom was fixed with $1 \%$ formalin for $15 \mathrm{~min}$. After one wash, Phase contrast images from 6 random microscopic fields were captured using Nikon Eclipse TE2000-S. Images acquired were captured using Focus Lite Version 2.88 software. Covered area of the thrombi seen in the images were calculated as described previously [7].

\subsection{Viability Assay}

TB-suspended platelets in a 96-well plate, with a final volume of $100 \mu \mathrm{l} /$ well, were incubated in $50 \mathrm{mM}$ of glutamine and exposed to UV-C, or in a lytic buffer and left non-treated for the desired time. The viability assay was assessed using $10 \mu \mathrm{l}$ of methylthiazoletetrazolium (MTT, Sigma). After 4 hours of incubation at $37^{\circ} \mathrm{C}$, the medium was replaced with $100 \mu \mathrm{l}$ of dimethylsulfoxide (DMSO, Sigma). The optical density was measured at $570 \mathrm{~nm}$ using a microplate spectrophotometer.

\subsection{Statistics}

Statistical analysis was carried out on raw data in SigmaPlot 9 using unpaired Student's t-test; a probability value, $p<0.05$, was considered statistically significant. Values are expressed as means with standard deviation and " $n$ " indicates the number of experiments.

\section{Result and Discussion}

Glutamine prevent the $U V-C$ induced activation of platelet. Washed human platelets were re-suspended in TB, supplemented with $1 \mathrm{~mm} \mathrm{Ca} 2+$ (Right panel, Figure 1. A.), PRP; not supplemented with calcium (Left panel, Figure 1. A.) were expose to UV-C in the presence or absence of glutamine. Addition of thrombin (positive control) produces multiple foci of thrombus formation in both panels. Similarly, platelets in their natural habitat i.e. plasma or TB washed platelets showed similar pattern of activation when exposed to UV-C, large mesh formation of induced platelets is noticeable in both, panels. Glutamine addition, however, abolishes this phenomenon in both the panels. Figure 1. A1, represents the statistical analysis of Figure 1. A, which shows the percentage of the covered area in the petri dish. Thrombin induced (positive control) and VU-C exposure lead to 50\% to $65 \%$ respectively, of the area covered area. Addition of glutamine, however, reversed the UV-C induced activation of platelets. These results demonstrated that glutamine could prevent the UV-C induced activation of platelets. Another intriguing answer we got from this experiment was the use of PRP or the TB washed platelets produces the same result.

In order to assess whether $50 \mathrm{mM}$ of glutamine could be toxic to cells, we used the viability assay (Figure 2) depicting the non-toxic effect of glutamine on platelets. In order to validate the data of our earlier publication [9] where we have demonstrated at length the effect of glutamine on mouse platelets, the viability assay of both (Human and Mouse) did not give much difference between the platelets under the same conditions. 


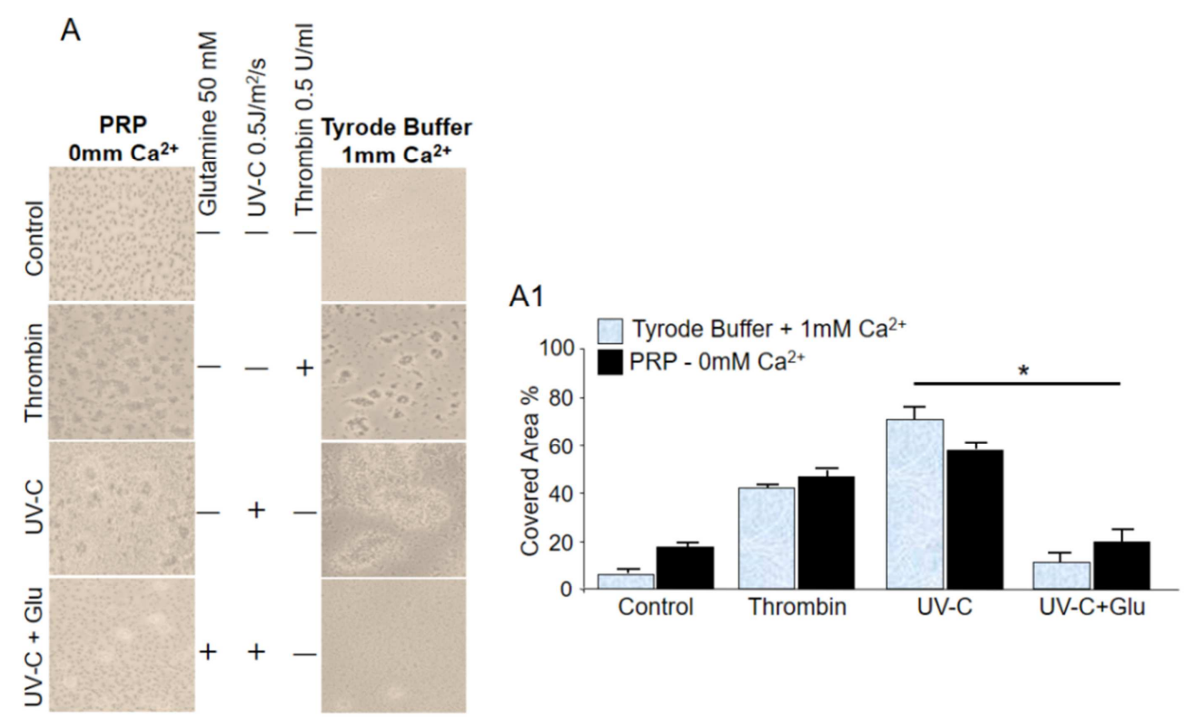

Figure 1. Glutamine can prevent the UV-C induced activation of human platelet: In vitro thrombus formation was assessed after exposing the human platelets to UV-C with or without $50 \mathrm{mM}$ glutamine; thrombin $0.5 U$ was used as positive control. $(A ; n=6)$. TB washed human platelets, supplemented with $1 \mathrm{mM} C a 2+$ were exposed to UV-C with or without 50mM Glutamine (right panel). Platelet Rich Plasma, (left panel) was similarly exposed. Significantly decreased, platelet covered area was observed with glutamine (A1), $* P<0.05$, measured by t test.

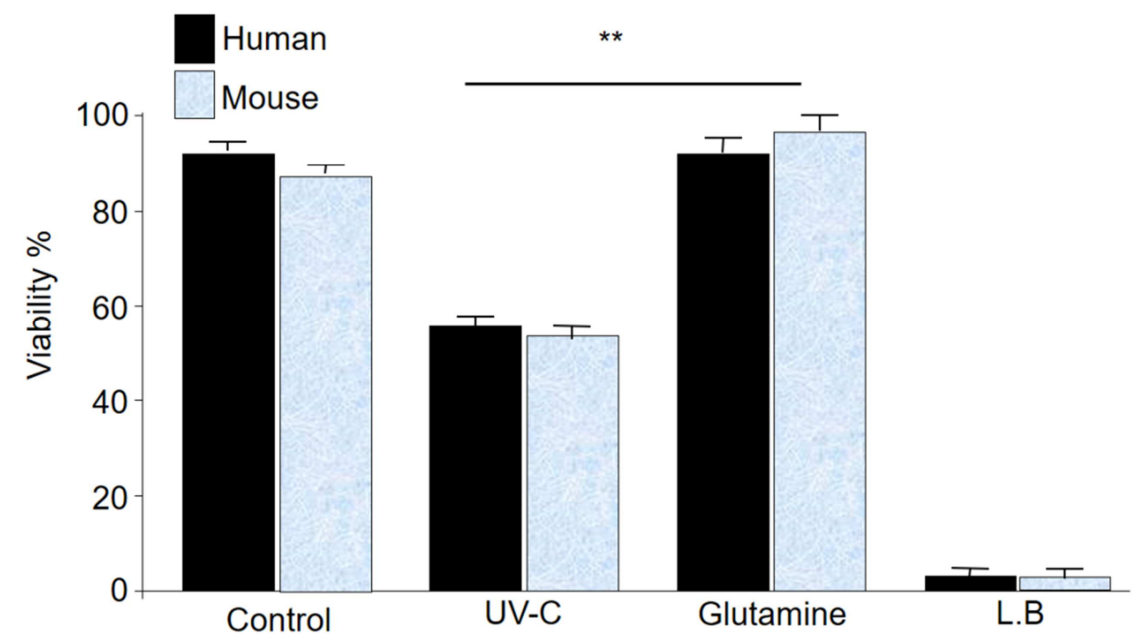

Figure 2. Glutamine toxicity effect was studied using CCK-8 kit (B; $n=4)$ of mouse and human platelets. The presence of glutamine was not toxic to platelets; however, viability was reduced to $50 \%$ with UV-C exposure, $* * P<0.05$. For control purpose, lysis buffer (LB) was used.

Platelet storage in the blood bank has been a critical issue. Frequent agitation, bacterial contamination and activation upon agitation result to the loss of their physiological function. Various measures have been employed to minimize the loss of physiological function of stored platelets concentrates. The use of broad spectrum wavelength, in the presence of a photosensitizer Riboflavin [10]; Mirasol Pathogen Reduction Technology System of ultraviolet (UV) light; UV-A (60\%), UV-B (100\%), and UV-C (20\%) was widely adopted all over the world as a standard procedure to prevent bacterial and viral contamination [11]. Furthermore, this technology has advanced to narrow band shortwave; "Theraflex", with specific wavelength $(254 \mathrm{~nm})$ of UV-C light and has become a valuable tool for safety and efficacy of platelets concentrate in the blood bank $[12,13]$. In some of the developed country this system has been adopted which expose the platelets as "one-shot" treatment and it does not require any additives, however, it's prolong exposure could severely produce malfunctioning of the platelets. In the developing or underdeveloped countries, Mirasol system still prevails [14-16]. Figure 3 sums up the differential properties of the different UV lights. Transfusion of viable platelets is critical. Different platelet concentrates storage solutions have been investigated; the use of a diluted autologous plasma, ViaCyte $^{\mathrm{TM}}$, and preservative solution, were all shown to preserve the ability of platelets to aggregate and secrete granule contents [17-19]. Theraflex ${ }^{\mathrm{TM}}$ has been widely employed for this purpose. A number of studies have been published showing its toxic effect, such as decreased thrombus formation property [20] and decreased collageninduced aggregation [12] with platelet exposure to UV-C. In our study, we used glutamine to nullify the toxic effect of Theraflex ${ }^{\mathrm{TM}}$. 


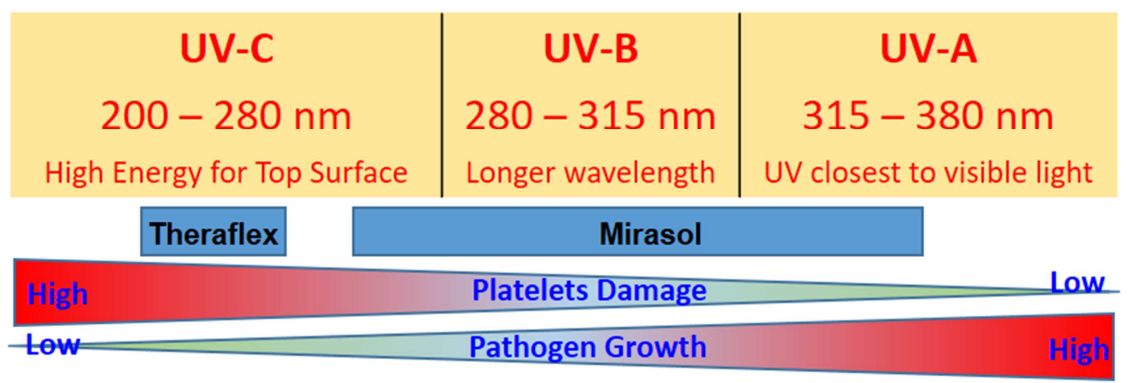

Figure 3. Differential properties of the different UV lights. UV-C narrow wavelength, high energy producing potential damage to platelets, however effective germicidal.

Glutamine after its transport [21] is actively metabolized in human platelets, representing a preferential mitochondrial oxidative substrate in these cells [22]. Furthermore, exogenous glutamine is metabolized by platelets to glutamate, aspartate, and $\mathrm{CO}_{2}$; with no lactate formation [12]. This make the platelets more viable, as they are less exposed to acidic environment.

\section{Conclusion}

There is not much focus on this topic in the literature. In this study, we demonstrated that the occurrence of physiological changes due to the exposure of platelet concentrates to UV-C could be alleviated by glutamine. We have delineated the different experiments in the mouse model, showing that glutamine preserves the physiological functions of platelets. In this study we have use the human platelets and have shown well preserve function of the platelets under the UV-C. However, a more in-depth study is required at the molecular level to assess the detailed pathway and how glutamine maintains the physiological functions of platelets. This study raises some questions. How and where does glutamine work in the anucleated cell to alleviate the toxic effect of UV-C? Does the presence of glutamine in the platelets have any direct impact on the growth of pathogens? These speculations leave this study subject to further metabolic studies on oxidative phosphorylation and glycolysis in the presence of UV-C and glutamine.

\section{Acknowledgements}

I am appreciative to Prof. Uh-Hyun Kim, head of Biochemistry Department in the medical section of Chonbuk National University, South Korea, who's endless efforts in the supervision and guidance of this work.

\section{References}

[1] Bashir S, Cookson P, Wiltshire M, Hawkins L, Sonoda L, Thomas S, et al. Pathogen inactivation of platelets using ultraviolet $\mathrm{C}$ light: effect on in vitro function and recovery and survival of platelets. Transfusion 2013; 53: 990-1000.

[2] Johnson L, Hyland R, Tan S, Tolksdorf F, Sumian C, Seltsam $\mathrm{A}$, et al. In vitro quality of platelets with low plasma carryover treated with ultraviolet $\mathrm{C}$ light for pathogen inactivation. Transfusion Med. Hemotherapy. 2016; 43: 190-197.

[3] Van Aelst B, Devloo R, Vandekerckhove P, Compernolle V, Feys HB. Ultraviolet $\mathrm{C}$ light pathogen inactivation treatment of platelet concentrates preserves integrin activation but affects thrombus formation kinetics on collagen in vitro. Transfusion 2015; 55: 2404-2414.

[4] De Jager TL, Cockrell AE, Du Plessis SS. Ultraviolet Light Induced Generation of Reactive Oxygen Species. In: Ahmad SI, editor. Ultraviolet Light in Human Health, Diseases and Environment. Cham: Springer International Publishing 2017; $15-23$.

[5] Britt Van Aelst, Rosalie Devloo, Philippe Vandekerckhove, et al. Ultraviolet $\mathrm{C}$ light pathogen inactivation treatment of platelet concentrates preserves integrin activation but affects thrombus formation kinetics on collagen in vitro. Transfusion 2015; 55: 2404-2414.

[6] Murphy S, Munoz S, Parry-Billings M, Newsholme E. Amino acid metabolism during platelet storage for transfusion. $\mathrm{Br} J$ Haematol 1992; 81: 585-590.

[7] Mushtaq M, Nam TS, Kim UH. Critical role for CD38mediated $\mathrm{Ca} 2+$ signaling in thrombin-induced procoagulant activity of mouse platelets and hemostasis. Journal of Biological Chemistry 2011; 15: 12952-12958.

[8] Seltsam, Axel \& Müller, Thomas. UVC Irradiation for Pathogen Reduction of Platelet Concentrates and Plasma. Transfusion medicine and hemotherapy 2011; 38: 43-54.

[9] Mazhar M, Kim UH. The Role of Glutamine in the Prevention of Ultraviolet-C-Induced Platelet Activation. Biochemistry Research International. 2020. DOI: 8853696, 7 Pages.

[10] Shirley Owusu-Ofori, Joseph Kusi, Alex Owusu-Ofori, Graham Freimanis, et al. Treatment of whole blood with riboflavin and UV light: impact on malaria parasite viability and whole blood storage. Shock 2014; 44: 33-38.

[11] Jerrold H. L, Matthew D. Neal, and Jay H. Herman. Bacterial contamination of platelets for transfusion: strategies for prevention. Critical Care 2018; 22: 271-278.

[12] Mohr H, Steil L, Gravemann U, Thiele T, Hammer E, Greinacher A, et al. A novel approach to pathogen reduction in platelet concentrates using short-wave ultraviolet light. Transfusion 2009; 49: 2612-2624.

[13] Seltsam A, Muller TH. UVC irradiation for pathogen reduction of platelet concentrates and plasma. Transfusion Med. Hemotherapy 2011; 38: 43-54. 
[14] Freimanis G. Investigating the Prevalence of Transfusion Transmission of Plasmodium within a Hyperendemic Blood Donation System. Transfusion 2013; 53: 1429-1441.

[15] Kabita C, Shamsuz Z, Rahul C, Surinder S, Shawn DK, Shalini T, et al. Evaluation of Mirasol pathogen reduction system by artificially contaminating platelet concentrates with Staphylococcus epidermidis: A pilot study from India. Asian $J$ Transfus Sci. 2016; 10: 127-131.

[16] Asa'ah N, Gabriel A, Emmanuel A, Claude T, Tazoacha A. Whole blood pathogen reduction technology and blood safety in sub-Saharan Africa: A systematic review with regional discussion. Afr J Lab Med. 2016; 5: 363.

[17] Terpstra FG et al. Potential and limitation of UVC irradiation for the inactivation of pathogens in platelet concentrates. Transfusion 2008; 48: 304-313.

[18] VandenBroeke T, Dumont LJ, Hunter S, Nixon J, Murphy S, Roger J, et al. Platelet storage solution effects on the accuracy of laboratory tests for platelet function: a multi-laboratory study. Vox Sanguinis 2004; 86: 183-188.

[19] Karnicki K, Johnson C, St Cyr J, Ericson D, Rao G. Platelet storage solution improves the in vitro function of preserved platelet concentrate. Vox Sanguinis 2003; 85: 262-266.

[20] Zhi L, Chi X, Vostal JG. In vitro and in vivo characterization of ultraviolet light $\mathrm{C}$-irradiated human platelets in a 2 event mouse model of transfusion. PLoS One 2013; 8: e79869.

[21] Valeria V, Elisabetta M, Marta F, Paola B. Glutamine transport and enzymatic activities involved in glutaminolysis in human platelets. Biochim Biophys Acta 1995; 1243: 43-48.

[22] Valeria V, Elisabetta M, Marta F, Paola B. Glutamine Utilization in Resting and Stimulated Platelets. The Journal of Biochemistry 1993; 114: 163-166. 